\title{
Digital Nomads and the Changing Work Life of Technology Entrepreneurs in Nigeria
}

\author{
Oluwaseun David Adepoju $^{1}$, Tolulope Omolara Agunbiade ${ }^{2}$ \\ ${ }^{1}$ Research Methods Faculty and Academic Librarian \\ African Leadership University, Kigali Rwanda \\ ${ }^{2}$ Entrepreneurship Faculty, African Leadership University, Kigali Rwanda
}

\begin{abstract}
Work life is changing and there is a new wave of work mode that is taking over the 21st century work space. Digital nomadicity has been an issue of discussion in the innovation space in the last one decade and the idea is becoming more and more accepted and explored across the world. Quite a number of research have been done on the emergence and lifestyle of digital nomads in different parts of the world but little or no empirical study has been done on the emergence of digital nomads in Nigeria and how this is influencing the regular 9-to5 work. This study explores digital nomads on the basis of their four digital nomads elements such as adventure and global travel, digital gig, gig work and nomadic work. This study serves as a reference to the emerging digital nomadicity in Nigeria and the changing landscape of work life for technology entrepreneurs.
\end{abstract}

\section{Introduction}

Technology has changed the work landscape in organizations and the idea of going to a physical office dedicated to a particular organization is becoming gradually obsolete. With the advancement in collaborative technologies and enterprise solution building skills, it has been observed that co-working spaces and innovation hubs are beginning to take over the future of offices and work. Most of the technology entrepreneurs prefer to work remotely on the move on in open innovation hubs without having to be necessarily be in the building of the organization they serve. Much more interesting is the fact that most technology entrepreneurs these days provide solutions to organization they have not even met in physically. This makes working easy and flexible for most of them. In the era where we call these aforementioned people digital nomads, it is very important to understand what fulfillment of purpose in terms of work life means to digital nomads since they no longer have to resume in a business dedicated office every single morning of their lives. In this study, insights are gained into the current perspectives of the general society on the real meaning of digital nomadism and the implications for the future of work in Nigeria. This study also $\mathrm{x}$ trays the challenges faced by digital nomads in Nigeria in terms of acceptance and cultural insinuations to hardwork and Sweaty Labor. Touches were made on the level of supposed financial prosperity of technology entrepreneurs as against their 9-5 counterparts. The study ends by summarizing the lessons and insights gotten from the whole analysis and suggestions were made for future research.

\section{Literature Review}

A number of public commentaries and opinions have been published globally on digital nomadism, however, solid research articles are still relatively scarce on this subject matter due to its gradual emergence in Nigeria. It is proud moment for the authors to note that this study is one of the foremost in Nigeria that is taking an academic research approach to studying digital nomadism [1, 4]. The explosive rise in the population of digital nomads was noted in the United states in earlier in 2018 when State of Independence America [3] discovered that 4.8 million people are already referring to themselves as independent workers or digital nomads. This unanimously pointed to the fact that many people are looking for freedom and independence in their work lives and they are finding the way put through digital nomadism. This growth in the number of digital nomads might possibly be an aftermath of tyranny and unfavourable treatment of employees by one man business bosses.

We cannot undermine the roles of technology in the maintenance of the digital nomad culture. Technology has remained the major driver of this culture and this has enabled digital nomads to create digital products using digital tools as they travel the world [4]. According to Nash et al. [2], "digital nomads engage with works that create digital goods using digital tools, what research has recently begun calling "digital work". Gleanings from the afore stated is indicative of the fact that digital nomads largely engage in digital works. This understanding can give us the ground to conclude that digital 
nomadism is directly intertwined with technology entrepreneurship. Since technology has broken down the physical barrier of work limitation, digital nomads can easily sit anywhere in the world and engage in digital works that directly serves any location in the world either cumulatively or real time service. The description of the content of the wok of digital nomads above should give us an insight into the broad categories these people fall into. Nash et., [2] noted that digital nomads falls largely into the categories of programmers, developers, designers, or content creators. We can further break these categories down into website designers, software engineers, data scientists, web app developers and creative content developers. This categorization of digital nomads is in resonance with the categories of the digital nomads interviewed in this study as each of them falls into one of the categories represented in the one stated above. It will not be an overstatement to note that digital nomads all around the world possess relatively similar skills that are basically digital and technological. In appreciating all the skills that skills that digital nomads possesses, Gandini [1] stated that digital nomads will be needed more in the digital society as they contribute to the digital economy. Their presence on the society has increased the capacities of the digital society to create more contents and businesses are able to reach more people with their services through the remote contributions of digital nomads. As they travel the world, they are becoming global citizens and they are acquiring knowledge of cultures and context which one way or the day influences their abilities to deliver premium services to clients and corporates. Every society must begin to measure and track the contributions of digital nomads to its economy. In Nigeria, efforts should be made by the government or some interested NGO's in studying the contribution of the digital technology ecosystem to the Nigeria GDP. In conclusion, this review has been able touch on rapid increase of digital nomads all around the world, the flexibility of their work, the content of their work, the skills they possess, their categories in professional and their contributions to the digital society and its economy.

\section{Methodology}

An expository research was conducted using qualitative research method of an interview type. Naked data was gathered through a methodological triangulation from two sources: analytics of Nigerian based popular digital nomad forums and a series of interview for 5 digital nomads who are originally Nigerians but working remotely in Nigeria or from other parts of the world either providing services for a Nigerian firm or other foreign firms. Their responses are subjected to critical analysis and interpretation in order to understand the current situation of digital nomadism in Nigeria and the impact it is having on technology ecosystem and work life generally.

\subsection{Analysis of Interviews and Forum Statements}

A series of questions were prepared as guided interview points for the randomly selected digital nomads in Nigeria. The following questions were asked in no particular order:

- Would you classify yourself as a digital nomad?

- Do you prefer to work from home/innovation hubs/co-working spaces or go to work (The organizational building)?

- Do you feel fulfilled in your work life working remotely?

- What are the advantages of working remotely as compared to working from the dedicated organizational building?

- What do you think is the future of office spaces in Nigeria?

On Techpoint.Africa, the biggest tech news and forum company in Nigeria, It was discovered that most of the forum articles on digital nomadism had no comments. Could it be that many people are not yet in tune with this new idea of remote working? For example, on a forum post titled "working nomads: VR for education and more", the below statement was made:

"Won't you go to work today?" If you have to answer this question or a different version of it frequently, especially with the suspicious glare that it typically comes with, then you probably belong to the league of remote workers. Well, in this part of the world, that could also make you some sort of alien a lot of people still do not understand that you can get the work done without leaving your home".

The statement above seems to be the experience of most digital nomads and remote worker in Nigeria. In this part of the world where people expect you to wake up in the morning and dress up for work, you are tagged lazy or fraudulent if you don't go to work when others are going. To corroborate this, Joel, a UI/UX developer in Ibadan Nigeria had this to say on this particular issue;

"I have been tagged an online fraudster in my neighborhood because I don't go to work when others in my house are going and I seem to be okay 
economically than all my neighbors. I was confronted many times in my community on what exactly I do for a living. If they see me outside at all, I am with my computer because I service all my clients remotely and I get paid well for my services. I am tagged a "yahooboy", the slogan for online fraudsters in Nigeria because of my nomadic workstyle".

As Joel recounted this experiences of his, it was glaring to me that many people are still not aware that the work culture they had always known has changed and it's not everyone who stays at home or uses a computer in a coffee shop commits fraudulent acts online. A lot of young men and women who are technology entrepreneurs in Nigeria now works from home or different parts of the world while serving their clients but many of them have been termed "fraudulent" because they do not seem to work the way other conventional people work.

In line with these thoughts, Tunde, a software developer in Akure, Ondo state Nigeria recounted how he was arrested by the men of the NIgerian Police force after being reported to be a fraudulent individual because of working with his laptop from a popular eatery in Akure city. Tunde had this to say:

"I was arrested on the 19th of May 2017 in an eatery in Akure because I had always worked from there. A staff member of the eatery reported me to the Nigerian Police force that I am a "yahooboy". I do not know when using a computer has become fraudulent? I was released after two days on interrogation and discovery that I was just a software developer who works remotely. The digital nomad culture is still very unpopular in most parts of Nigeria and it is very sad that we face the fire for this. I do hope that one day, digital nomadism will become very popular and people would not have to judge us for the kind of work life we have embraced".

Tunde's account is another indication that digital nomadism is still very unpopular in some parts of Nigeria. Most digital nomads still lives in the risk of being misrepresented and seen as an online criminal. The field of digital nomadism is still evolving and it will become relatively popular in all the parts of the country in the coming days.

An interview with Abiodun, a 29 years old blockchain and cryptocurrency portfolio trader in Ibadan Oyo state Nigeria, he noted that many people were surprised when he left is 9 to 5 job in a teaching hospital in Nigeria to fully concentrate on his cryptocurrency investment portfolio. Abiodun had this to say:

"I was getting choked with my 9-5 job because I was not having enough time to do what I am really passionate about. My remote cryptocurrency trading started paying me far more than my normal 9 to 5 job and I eventually made a decision to pull out. I was under the verbal barrels of my colleagues and superiors who thought I was leaving to job to become an online fraudster because I was actually driving a car better than most of my bosses. I think digital entrepreneurship comes with this financial prosperity that seems impossible to a lame mind. They just feel your level of prosperity financially should commensurate with your sweat. Note it, we as digital entrepreneurs also sweat,but most times intellectual sweat".

Abiodun gave a new insight into the discussion as he noted that digital entrepreneurs are somewhat more financially successful than most people on the 9-5 payroll. This gives a literary validity to the point of Joel, when he said "I seemed to be economically successful than some of my colleagues who do regular jobs".

In a conversation with another digital nomad who was a fellow of the first cohort of Andela Nigeria, he noted that he has been in Spain for a month working for two clients in the United States. He said;

"I have been working remotely to service two companies in the USA while I am enjoying a vacation in Spain. I go scuba diving in the morning and return to my hotel room in the midday to continue coding. I feel I am living my full life because I get to combine work and fun without having to be under unnecessary pressures from any boss. I am the boss of my own and proactiveness is my strength. It is so amazing that you could enjoy the ambience of life and work at the same time. With digital nomadism, there is no need for a special vacation in the year as everyday is vacation and everyday is work at the same time".

Wilson brought a new insight into what we can describe as vacate while you work. He seemed very enthusiastic about his work life as he noted the level of fulfilment he has working and vacating at the same time. He does not have to worry about taking a vacation after a rough work life. Both work and relaxation goes hand in hand and this possibly increases productivity. We should all be digital nomads I guess.

Adetola, an android app developer in Lagos state Nigeria brought yet another deep insight into the whole conversation by differentiating what it means to be a hard worker and a smart worker. Adetola noted that the society has not yet come to term with what it is to be a smart worker as against the popular societal slogan of hard work. He said: 
"I literally made what my father made in his 35 years of public service in just 5 years because of technology and technology business that provide services to clients on a global scale. Technology made my work smart and I did not need to report to anybody in the morning before I work from the corner of my room. I sleep when I want and eat when I want while I am still working. Hard Work is good but smartwork is better in the technology centered society".

The researchers are of the opinion that it is not unlikely that the greatest determinant of being a digital nomad is how smart an individual is in handling several task with efficiency and precision. The results summary are briefly listed below:

1. Digital nomadicity is still relatively unpopular in most parts of Nigeria

2. It seems to be the future of work option that combines both work and vacation at the same time

3. There is somewhat an economic and financial prosperity that comes with being a digital nomad as discovered form the responses.

4. Digital nomads and digital entrepreneurs face many issues with anti-crime commissions and the Nigerian Police force because of misrepresentation of identity.

5. Most digital nomads may never feel comfortable with 9-5 job life after being successful as a digital nomad.

6. Digital nomadicity is more popular in Lagos state Nigeria than in any other part of Nigeria.

The digital nomadism in Nigeria is evolving and the future potentials seem great for the Nigeria Innovation Ecosystem. Work is changing and working conditions are also drastically changing. We may not have seen the full endpoint of future of work in Nigeria but the current situation in digital nomadicity is giving us an insight into what we should expect in the next 5 years. we are most likely to have more smart workers and more people vacating the conventional offices. In this study, we have been able to make the aforementioned projections from the interview sessions from 5 digital nomads and technology entrepreneurs in Nigeria.

\section{Conclusion}

This study is regarded as an expository perspective to digital nomadism in Nigeria and not in any way conclusive yet. Digital nomadism is still an evolving subject in the Nigerian Innovation
Ecosystem. However, a number of technology entrepreneurs in Nigeria are already getting the dividends of these emerging field of interest. This study was able to analyze the current situation in Nigeria by interviewing five digital nomads and an evaluation of some digital nomads forum in Nigeria. Insights were gained and projections were made on the future of work in Nigeria and the trends of evolution of digital nomadism in Nigeria in next 5 years.

\section{Reference}

[1] Gandini, A., (2016) The Reputation Economy: Understanding Knowledge Work in Digital Society. Springer, London

[2] Nash, C., Jarrahi, M. H., Sutherland, W., and Phillips, G., (2018). Digital nomads beyond the buzz -word: De -fining digital nomadic work and use of digital technologies. Lecture Notes in Computer Science (Including Subseries Lecture Notes in Artificial Intelligence and Lecture Notes in Bioinformatics), 10766 LNCS (February), 207-217. https://doi.org/ 10.1007/978-3-319-78105-1_25

[3] Independence, A. S. (2018). Digital Nomadism: A Rising Trend, 2017-2019.

[4] Spinks, R., (2015) Meet the 'digital nomads' who travel the world in search of fast Wi-Fi. Guardian 2017, (2015). 